\section{Sepsis in der Geburtshilfe: Score erlaubt Vorhersage schwerer Verläufe}

Albright CM et al. Internal validation of the sepsis in obstetrics score to identify risk of morbidity from sepsis in pregnancy. Obstet Gynecol 2017; 130: 747-755. doi:10.1097/AOG.0000000000002260

Viele etablierte klinische Scores zur Beurteilung des Schweregrads einer Sepsis berücksichtigen nicht die typischen Veränderungen während der Gravidität, beispielsweise die physiologische Tachykardie. Um diese Lücke zu füllen, haben US-Wissenschaftler anhand einer retrospektiven Kohorte den schwangerschaftsspezifischen „Sepsis in Obstetrics Score" entwickelt. $\mathrm{Er}$ wurde nun an einem prospektiven Kollektiv validiert.

In den „Sepsis in Obstetrics Score“ fließen die Körpertemperatur, der systolische Blutdruck, die Herz- und Atemfrequenz, die periphere Sauerstoffsättigung, die Leukozytenzahl, der Anteil unreifer Neutrophiler sowie die Laktatkonzentration ein. In der retrospektiven Entwicklungskohorte hatte sich ein Score von 6 Punkten zur Vorhersage einer intensivmedizinischen Behandlungspflichtigkeit der Patientinnen als optimal erwiesen. Die prospektive Validierung erfolgte an einem Kollektiv von 1250 Müttern, die sich während der Schwangerschaft bzw. bis zu 2 Wochen nach der Geburt aufgrund eines SIRS (Systemic Inflammatory Response
Syndrome) in der Notaufnahme des "Women and Infants Hospital“ in Providence/Rhode Island vorstellten. Die Berechnung des Scores erfolgte anhand der aufgezeichneten Vitalparameter und der Laborbefunde der Patientinnen. Der individuelle Punktwert war zum Zeitpunkt der Erstversorgung unbekannt. Primärer Studienendpunkt war die Intensivpflichtigkeit aufgrund einer Sepsis innerhalb von 48 Stunden. Die sekundären Outcome-Parameter umfassten die Notwendigkeit einer telemetrischen Überwachung, die Dauer des Klinikaufenthalts, die Blutkultur- und Influenzatestergebnisse sowie die Zeit bis zur Einleitung einer antibiotischen Therapie. Ferner wurde der prädiktive Nutzen des „Sepsis in Obstetrics Score“ mit der Performance weiterer, nicht schwangerschaftsspezifischer Scores verglichen.

\section{Ergebnisse}

Bei 425 Frauen (34\%) wurde die Diagnose "Sepsis“ gestellt, da bei ihnen ein klinischer Infektionsverdacht bestand bzw. eine Infektion nachgewiesen werden konnte. In 14 dieser Fälle war eine Behandlung auf der Intensivstation erforderlich und weitere 45 Patientinnen mussten telemetrisch überwacht werden. Zehn der intensivmedizinisch behandelten Patientinnen $(71,4 \%)$ waren Schwangere. Die auf die Intensivstation aufgenommenen Frauen litten am häufigsten an einer Pyelonephritis, Pneumonie oder Influenza. Die Patientinnen mit einem Score $\geq 6$ wiesen im Vergleich zu Patientinnen mit einem 
niedrigeren Punktwert einen signifikant höheren Body-Mass-Index auf und hatten signifikant häufiger eine Pyelonephritis und seltener eine Gastroenteritis. Weitere Parameter, die bei einem Score $\geq 6$ signifikant häufiger auftraten, waren eine fetale Tachykardie, eine stationäre Aufnahme, eine Aufnahme auf die Intensivstation sowie eine telemetrische Überwachung. Ferner war bei hohen Punktwerten der Klinikaufenthalt länger, es wurden häufiger Blutkulturen abgenommen, die zudem häufiger positiv waren, und es wurden häufiger und schneller Antibiotika verordnet. Die Analyse der ROC-(Receiver-Operating-Characteristic-)Kurve bezüglich der Prädiktion der Intensivpflichtigkeit ergab für den „Sepsis in Obstetrics Score“ eine AUC (Area Under the Curve) von 0,85 (95\%-KI 0,76-0,95). Bei einem Cut-off von 6 errechnete sich für den Score eine Sensitivität von 64\% (95\%-KI 35,1-87,2), eine Spezifität von 88\% (95\%-KI 85-90,6), ein positiver Vorhersagewert von $15 \%$ (95\%-KI 7,1-26,6) sowie ein negativer Vorhersagewert von 98,6\% (95\%-KI 96,899,6). Anders als von den Autoren erwartet, zeigten 2 der etablierten Scoring-Systeme im Studienkollektiv eine vergleichbare prädiktive Performance wie der schwangerschaftsspezifische Sepsis-Score.

FAZIT

Geburtshilfliche Patientinnen, die aufgrund eines septischen Krankheitsbilds einer intensivmedizinischen Behandlung bedürfen, so die Autoren, lassen sich mithilfe des „Sepsis in Obstetrics Score“ zuverlässig identifizieren und einer zielgerichteten Behandlung zuführen. Andererseits kann aufgrund des hohen negativen Vorhersagewerts bei einem Score $<6$ von einem minimalen Risiko für eine klinische Verschlechterung ausgegangen werden. So können unnötige Interventionen vermieden werden.

Dr. med. Judith Lorenz, Künzell 